\title{
Allergologische Raritäten aus dem erotischen Spannungsfeld
}

_ Vom Kanarienvogel bis zum geplatzten Kondom, vom liebestötenden Parfum bis zum erigierten Unterarm - die „Köln Session“ hatte einiges zu bieten. So war beispielsweise der Genuss von Mozarts Zauberflöte in der Kölner Oper für ein frisch verliebtes Seniorenpaar nur von begrenzter Dauer, da der Mann, der unter allergischem Asthma litt, noch vor der Pause einen Asthmaanfall mit Hustenreiz und Atemnot bekam. Das erst zu Hause verfügbare Asthmaspray ließ zwar ein durchaus intensives Näherkommen zu, zeigte aber nur

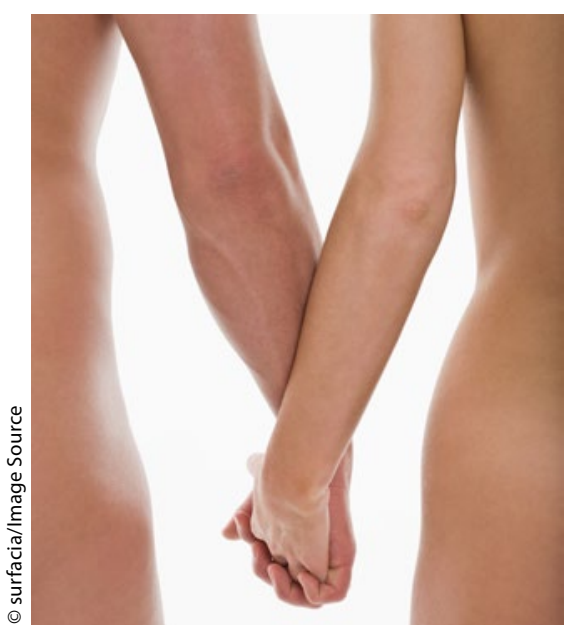

vorübergehend Wirkung. Als Ursache dafür will Dr. Norbert Mülleneisen, Leverkusen, den Inhaltsstoff eines durchaus hochpreisigen Parfums ausgemacht haben - nämlich ein Analdrüsensekret der Zibetkatze.

Zieht man die Lehren aus einer weiteren von Mülleneisen vorgestellten Kasuistik, sollte man männlichen Patienten nach einem Pricktest möglicherweise den Rat geben, in direkter zeitlicher Folge auf die Einnahme von Viagra und Co lieber zu verzichten. Bei dem betreffenden Patienten war es zwar nach der späteren Einnahme von Tadalafil sozusagen plangemäß zu einer heftigen Erektion gekommen - nur an der falschen Stelle, nämlich am Unterarm. Tatsächlich werden Angioödeme, Hautrötungen und Überempfindlichkeitsreaktionen vom Hersteller als sehr häufige Nebeneffekte dieses PDE-Hemmers angegeben.

Als einem in die Jahre gekommenen frühpensionierten internistischen Chefarzt im Zuge einer neuen Liebschaft im wahrsten Sinne des Wortes mehr und mehr die Luft weg blieb, hatten Bochumer Arbeitsmediziner Detektivarbeit zu leisten, bis sie dem Grund des Übels auf die Spur kamen. Als Täter überführt werden konnten Kanarienvogel-Antigene. Die neue Liebe war die Ex eines Kanarienvogelzüchters und der verliebte Chefarzt war an einer exogen-allergischen Alveolitis erkrankt.

Über eine sehr ungewöhnliche Variante der spezifischen Immuntherapie (SIT) hat Prof. Dr. Knut Brockow, TU München, anhand der Fallgeschichte einer Patientin mit Seminalplasmaallergie berichtet. Bei dieser seltenen Erkrankung kommt es wenige Minuten nach ungeschütztem Geschlechtsverkehr zu einer mit vaginalem Juckreiz und Brennen einhergehenden allergischen Sofortreaktion, die mitunter lebensbedrohliche Züge (Urtikaria, Angioödem, Schock) annehmen kann. Als verantwortliches Allergen vermutet man ein dem Prostata-spezifischen Antigen (PSA) zuzuordnendes Protein. Eine in München vorgenommene Hyposensibilisierung mit aufgereinigtem PSA erwies sich - so das Ergebnis von bis zum Koitus führenden intravaginalen Provokationsversuchen in steigender Dosierung - zwar als prinzipiell erfolgreich, bisherige Hyposensibilisierungsversuche mit Seminalplasma waren aber nicht dauerhaft. Um das immunologische Gedächtnis in der notwendigen Weise zu unterstützen, bedürfe es offenbar einer so hohen Kohabitationsfrequenz, dass betroffene Paare auf Dauer überfordert wären. Dr. Ludger Riem

AllergoCase: The Köln Session: Allergologie im Spannungsfeld der Geschlechter

\section{Können Allergien zu Schwerhörigkeit führen?}

— Dass Allergien Sinnesleistungen wie Riechen und Schmecken beeinträchtigen können, ist bekannt. Die Rolle, die allergische Entzündungsvorgänge bei verschiedenen Formen der Schwerhörigkeit spielen können, wird dagegen häufig unterschätzt. Eine Erkrankung des Mittelohrs, die mit einer Schallleitungsschwerhörigkeit einhergeht, ist das Seromukotympanon. Nahezu jedes Kind leidet einmal an einem Paukenerguss. Bei rund einem Drittel kommt es allerdings zu rezidivierenden oder prolongierten Verläufen, berichtete Prof. Dr. Michael Damm, HNO-Universitätsklinik Köln. In diesen Fällen ist eine erweiterte Abklärung sinnvoll [Damm M et al. HNO 2013; 61:843-8].

Wie auch die aktuelle Leitlinie festhält, kann neben vielen anderen Ursachen eine aller- gische Verschwellung der Tubenostien ein Seromukotympanon begünstigen [Lautermann J et al. HNO 2012;60:540-4]. Nicht nur das lymphatische Gewebe im Nasenrachenraum, sondern auch die Tuba auditiva sowie die Mittelohrschleimhaut können Angriffspunkte allergischer Entzündungsvorgänge sein, erklärte Damm.

Als Auslöser, die bei der Ausbildung beziehungsweise Persistenz eines Seromukotympanon eine Rolle spielen können, kommen sowohl Aero- als auch Nahrungsmittelallergene in Betracht. Damm riet, insbesondere bei rezidivierenden oder prolongierten Episoden eine Allergie in die diagnostischen Überlegungen miteinzubeziehen und gegebenenfalls eine ergänzende allergologische Behandlung in die Wege zu leiten.
Auch bei der Innenohrschwerhörigkeit können Allergien in Einzelfällen eine Rolle spielen, sagte Damm. So gibt es Hinweise darauf, dass Allergien einen Morbus Menière triggern können [Takeda T et al. Otol Neurotol 2012;33:886-90]. Studiendaten weisen zudem darauf hin, dass bei Patienten mit Morbus Menière die Prävalenz sowohl von Inhalations- als auch von Nahrungsmittelallergien erhöht ist und sich unter einer antiallergischen Therapie bzw. Allergenkarenz auch die Symptomatik bessert [Derebery $\mathrm{MJ}$ et al. Curr Allergy Asthma Rep 2007;7:451-6]. Bei Patienten mit Morbus Menière könne daher auch eine allergologische Abklärung und gegebenenfalls ein Therapieversuch mit Antiallergika hilfreich sein, so Damm.

Angelika Bauer-Delto

AllergoCompact: Allergie und Sinne 